\title{
Order/disorder gradients of $n$-alkanethiols on gold
}

\author{
Magnus Lestelius a , Isak Engquist a, Pentti Tengvall a, Manoj K. Chaudhury ${ }^{\text {b }}$, \\ Bo Liedberg ${ }^{\mathrm{a}, *}$ \\ a Molecular Films and Surface Analysis Group, Laboratory of Applied Physics, Linköping University, SE-581 83 Linköping, Sweden \\ ${ }^{\mathrm{b}}$ Department of Chemical Engineering, Lehigh University, Bethlehem, PA 18015-4791, USA
}

Accepted 11 August 1998

\begin{abstract}
This paper explores the interfacial properties of one-dimensional molecular gradients of alkanethiols $\left(\mathrm{HS}-\left(\mathrm{CH}_{2}\right)_{n}-\right.$ $\mathrm{X}$ ) on gold. The kinetics and thermodynamics of monolayer formation are important issues for these types of mixed molecular assemblies. The influence of chain length difference on the contact angles with hexadecane (HD), $\theta_{\mathrm{a}}$ and $\theta_{\mathrm{r}}$, and the hysteresis, has been studied by employing alkanethiols $\mathrm{HS}-\left(\mathrm{CH}_{2}\right)_{n}-\mathrm{CH}_{3}$, with $n=9,11,13,15$ and 17 , in the preparation of the self-assembled monolayers (SAM) gradients. The contact angles with hexadecane, at the very extreme ends of the gradients, show characteristic values of a highly ordered $\mathrm{CH}_{3}$-like assembly: $\theta_{\mathrm{a}}=45-50^{\circ}$. In the middle of the gradients $\theta_{\text {a }}$ drops noticeably and exhibits values representative for $\mathrm{CH}_{2}$-like polymethylenes, $\theta_{\mathrm{a}}=20-30^{\circ}$, indicating a substantial disordering of the protruding chains of the longer component in the gradient assembly. As expected, the exposure of $\mathrm{CH}_{2}$-groups to the probing liquid increases with increasing differential chain length of the two $n$-alkanethiol used, in this case eight methylene units. However, the contact angles always display a non-zero value which means that even at a chain length difference of eight methylene units there is a substantial exposure of methyl $\left(\mathrm{CH}_{3}\right)$ groups to the probing liquid. With infrared reflection-absorption spectroscopy (IRAS) we have monitored the structural behavior of the polymethylene chains along the gradient. We find complementary evidence for disordered chains in the gradient region, and the IRAS results correlate well with the contact angle measurements. (C) 1999 Elsevier Science B.V. All rights reserved.
\end{abstract}

Keywords: Gold; Interfacial properties; Molecular gradients

\section{Introduction}

With an existing methodology to prepare twocomponent molecular gradients of self-assembled monolayers (SAMs) of alkanethiolates on gold

\footnotetext{
* Corresponding author.

E-mail address: bol@ifm.liu.se (B. Liedberg)
}

$[1,2]$ we want to address a number of critical issues of relevance for the development of novel biomaterials [3,4] and biosensing interfaces [5-7]. We are also interested in gaining a deeper fundamental knowledge about the interfacial properties of these assemblies [8]. For these gradients, two important monolayer properties immediately become available for variation, the density of protruding chains bearing specific functional groups 


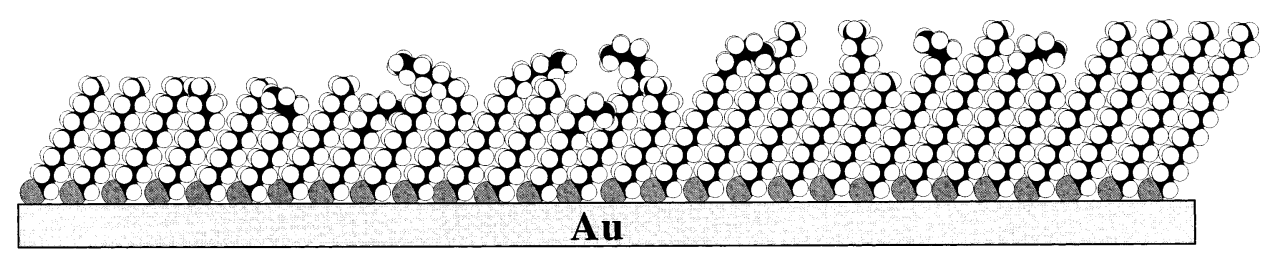

Fig. 1. Schematic picture of a order/disorder gradient $\left(\mathrm{HS}\left(\mathrm{CH}_{2}\right)_{11} \mathrm{CH}_{3} / \mathrm{HS}\left(\mathrm{CH}_{2}\right)_{17} \mathrm{CH}_{3}(\mathrm{Cl2} / \mathrm{C} 18)\right)$ assembled on gold.

and their mobility. For example, the possibility of a ligand to bind to an immobilized receptor in a biological recognition event depends to a large extent on the density and accessibility (mobility) of the receptor. The density and mobility of recognition centres are therefore important design parameters during development of biosensing interfaces. The impact of surface chemistry on protein adsorption also has been demonstrated on several occasions using various types of SAMs, not just on gold but on $\mathrm{Si}$, Ti and glass as well [7,10-13]. Prime et al., for example, investigated the protein resistance of flexible long-chain oligo(ethylene) oxide modified SAMs $[9,10]$. Recently, microscale gradients have been prepared using photoactivatable biomolecules for cell adhesion and cell migration experiments [14,15].

As mentioned above, our interests are not only focused on the applications of these gradients, but also on the mechanisms of assembly formation and microstructure. A long standing matter of concern is whether phase segregation occurs when two differently terminated molecules are mixed on the surface. In the case of longer versus shorter alkyl chains it is believed that thermodynamics play a major role for the phase behavior [16,17]. Bain et al. studied a series of mixed SAMs from $\mathrm{HS}-\left(\mathrm{CH}_{2}\right)_{11}-\mathrm{CH}_{3} / \mathrm{HS}-$ $\left(\mathrm{CH}_{2}\right)_{n}-\mathrm{CH}_{3}(n=15,21)$ adsorbed from ethanol and isooctane [18]. Using contact angle measurements, ellipsometry, and XPS they concluded that macroscopic islands do not form in these monolayers. Rather, if phase segregation occurs the domain size would be on the nanometer scale. A recent scanning tunneling microscopy (STM) study has shown that methyl- and esterterminated SAMs form domains a few nanome- ters in diameter [19]. In our study, the processes of exchange of alkanethiols between the surface and the solution [20], and the amount of intermixing in the diffusion matrix before/under adsorption are important events which we believe determine the composition and phase behavior of the gradient assemblies.

We have used water and hexadecane to measure contact angles since they probe different interactions, polar and dispersive [21]. The order/ disorder gradients present various amounts of $\mathrm{CH}_{3}$ and $\mathrm{CH}_{2}$ groups at the interface (Fig. 1). The advancing contact angle with water varies only little between $\mathrm{CH}_{3}$ and $\mathrm{CH}_{2}$ groups, $\theta\left(\mathrm{CH}_{3}\right)_{\mathrm{a}} \sim 110^{\circ}$ and $\theta\left(\mathrm{CH}_{2}\right)_{\mathrm{a}} \sim 102^{\circ}$, which makes it less useful for probing hydrocarbon chain order/disorder phenomena. On the other hand, water contact angle measurements are very sensitive to contamination, e.g. from the polysaccharide matrix used in the preparation. In contrast, hexadecane displays good selectivity between $\mathrm{CH}_{3}$ and $\mathrm{CH}_{2}$ groups, $\theta\left(\mathrm{CH}_{3}\right)_{\mathrm{a}} \sim 45-$ $50^{\circ}$ and $\theta\left(\mathrm{CH}_{2}\right)_{\mathrm{a}} \sim 0$ [22]. Thus, hexadecane contact angles can provide information about the fraction of exposed methylene groups. It can also give hints to whether the components in the gradient SAMs phase segregate or not. Even at the largest difference in chain length, eight methylene groups, the contact angle of hexadecane does not reach zero at any point along the gradient. From the present study it is not completely clear if this depends on the incapability of the longer alkanethiolates to fold over and completely screen the $\mathrm{CH}_{3}$ portion of shorter ones, and hence only expose $\mathrm{CH}_{2}$ groups, or if segregation occurs on the microscopic level to present both methyl- and methylene groups to the vacuum interface even under the best mixing conditions in the monolayer. 
IRAS spectra exhibit peak positions at the far ends of the gradient assemblies that indicate ordered alkyl chains in the all-trans configuration. When entering the mixing region of the gradients, the $\mathrm{d}^{+}$(symmetric $\mathrm{CH}_{2}$ stretch) and $\mathrm{d}^{-}$(asymmetric $\mathrm{CH}_{2}$ stretch) peaks broaden and shift upwards indicating an increasing amount of disordered gauche-rich chains. This behavior was correlated and compared with contact angle measurements made on identically prepared gold substrates.

\section{Materials and methods}

\subsection{Gold films}

Two types of gold substrates have been used in this study, sputter-deposited gold on glass and electron beam evaporated gold on silicon. On pre-cut glass $\left(40 \times 8 \mathrm{~mm}^{2}\right)$ a $1 \mathrm{~nm}$ thick adhesion layer of chromium and then $200 \mathrm{~nm}$ of gold were sputter-deposited (base pressure $\sim 10^{-6}$ mbar). This substrate type was used for contact angle measurements and ellipsometry. A second type of gold substrate was used for contact angle and IRAS measurements. $200 \mathrm{~nm}$ of gold with an adhesion layer of $1 \mathrm{~nm}$ titanium were electron beam evaporated onto silicon $\left(30 \times 20 \mathrm{~mm}^{2}\right)$ pieces in a UHV chamber (base pressure $<4 \times$ $10^{-9}$ mbar) [23]. The so-prepared gold films were dismounted and stored in Petri dishes until use.

\subsection{Chemicals}

The $n$-alkanethiols of $\mathrm{HS}-\left(\mathrm{CH}_{2}\right)_{n}-\mathrm{CH}_{3}, n=9$, 11,15 and 17 were obtained from Fluka, Buchs of purity $>95 \%$, and from Lancaster, Morecambe, $(n=13)$, purity $>95 \%$. Ethyl alcohol, 95\% from Kemetyl, Stockholm was used as solvent and for rinsing. The water used was taken from a MilliQ water purification system (low organic content $<5$ $\mathrm{ppb}$ and high resistivity $>18 \mathrm{M} \Omega \mathrm{cm}$ ). The diffusion medium was a hydroxypropylated and crosslinked dextran, Sephadex LH20 (Pharmacia Biotech AB, Uppsala). This matrix material can be handled in both water and organic solvents.

\subsection{Gradient preparation}

An elaborate and rigorous protocol had to be developed to obtain reproducible and clean monolayer gradients. The gold substrates were cleaned in a solution (TL1) of 5 parts $\mathrm{H}_{2} \mathrm{O}$ (MilliQ), one part $\mathrm{H}_{2} \mathrm{O}_{2}$ (Merck, 30\%) and one part $\mathrm{NH}_{4} \mathrm{OH}$ (Merck, $25 \%$ ) at $80^{\circ} \mathrm{C}$ for $10 \mathrm{~min}$ and rinsed with copious amounts of water. The surfaces were then stored in poly(styrene) Petri dishes in laboratory atmosphere overnight. It has proven necessary to expose the gold substrates to laboratory atmosphere for at least a day to render them hydrophobic in order to prevent the polysaccharide matrix adhering to the surface [1]. Duran glass Petri dishes were cleaned in the TL1 solution and dried with nitrogen. The gold substrates were put in the bottom of the glass Petri dishes and 4.5 $\mathrm{g}$ of the Sephadex gel was distributed on top of the surfaces and saturated with $16 \mathrm{~g}$ of ethanol, which induced a swelling of the diffusion matrix. The Petri dish had to be gently shaken to evenly distribute the mixture in the whole dish. The excess of ethanol was allowed to evaporate (ca. $40-50 \mathrm{~min}$ ) to yield a final mixture of $3.3 \mathrm{ml}$ EtOH $g^{-1}$ Sephadex. Glass filters with pore size ca. $100-150 \mu \mathrm{m}$, cleaned in the TL1 solution and dried in an oven $\left(150^{\circ} \mathrm{C}\right.$, overnight), were carefully and gently pressed into the matrix at the ends of the surfaces. $600 \mu \mathrm{l}$ of each thiol solution ( $2 \mathrm{mM}$ in ethanol) in the diffusion pair was uniformly pipetted into the two glass filters. The lid of the Petri dish was put in place and sealed with Parafilm. The cross diffusion and adsorption process was allowed to continue for ca. $65 \mathrm{~h}$ before interruption, unless otherwise stated. The surfaces were picked up from the thiol/Sephadexmatrix and briskly rinsed with water and ethanol. Following this, was an elaborate cleaning procedure where the surfaces were ultrasonicated in water and ethanol. The solvents, in which the ultrasonication (10 $\mathrm{min})$ was performed, were switched back and forth between water and ethanol four times. Between each ultrasonication, the gradients were rinsed in EtOH and water several times, and the solvents were removed with an aspirator pump. This elaborate rinsing proce- 
dure was necessary in order to remove the polysaccharide matrix completely from the sample surface.

\subsection{Contact angle measurements}

Advancing $\left(\theta_{\mathrm{a}}\right)$ and receding $\left(\theta_{\mathrm{r}}\right)$ contact angles were measured in air with a Ramé-Hart NRL Model 100 goniometer (Ramé-Hart, NJ). The sample stage had been modified with a micrometer translational stage (Newport, CA) to give motion in the direction perpendicular to the line of sight of the goniometer microscope. The contact angle was measured by suspending a large drop $(\varnothing \geq 3 \mathrm{~mm})$ from the micrometer syringe (Gilmont, IL) without using a needle. In this way the drop could be dragged over the surface. A new set of $\theta_{\mathrm{a}}, \theta_{\mathrm{r}}$-values was measured every $\mathrm{mm}$. $\theta_{\mathrm{a}}$ was measured by moving the stage $1 \mathrm{~mm}$ forward and halt it to record the value, and $\theta_{\mathrm{r}}$ was measured by moving the stage forward an additional $0.5 \mathrm{~mm}$ and back $0.5 \mathrm{~mm}$ again to read the value. Deionized and purified (MilliQ) water and hexadecane (Fluka, >98\%), chromatographed over alumina (Merck, Brockman I), were utilized as the probing liquids. The contact angle could be monitored with a precision of $\pm 1^{\circ}$.

\subsection{Ellipsometry}

Single wavelength ellipsometry was performed on the gold substrates and the SAMs to determine thicknesses. The measurements were made using a Rudolph Research AutoEl III, equipped with a HeNe laser $(\lambda=632.8 \mathrm{~nm})$ and with an angle of incidence of $70^{\circ}$. The sample stage was equipped with a stepper motor for computer controlled motion. Values of $\Delta$ and $\psi$ were collected for clean gold substrates (radio-frequency plasma cleaned and cleaned in the TL1 solution) and for the monolayer modified gold. A three phase (ambient/organic film/gold substrate) parallel slab model was employed for evaluation of the thickness of the monolayer film, where the organic phase was assumed to be isotropic with a refractive index of $N=1.50+i 0[1,24]$.

\subsection{Infrared measurements in $U H V$}

The infrared measurements were carried out in a UHV system [25] where a base pressure of $2 \times 10^{-9}$ mbar or lower was maintained throughout the experiments. The UHV system was connected to a Bruker IFS 113v Fourier transform infrared spectrometer working under mild vacuum ( $<10$ mbar). The detection was achieved with a liquid nitrogen cooled MCT detector, mechanically connected to the UHV system. The IR beam was focused at the sample with $f / 16$ transfer optics at angle of incidence of $82^{\circ}$ with respect to the surface normal $[25,26]$. The in-house customized sample holder gave an effective measurement length of $22 \mathrm{~mm}$ over the gradient surfaces (the intensity at the ends was only reduced by $2 \%$ as compared to the average intensity of the middle). A total of $90 \%$ of the incident light was found within a spot size of 4 $\mathrm{mm}$ in diameter. 500 interferogram cans were collected at a resolution of $2 \mathrm{~cm}^{-1}$. Single beam spectra were obtained by measuring the entire gradient sample and then repeating the measurement again after sputter cleaning. IRAS spectra are displayed as $-\log \left(R / R_{0}\right)$ where $R$ and $R_{0}$ are the reflectivity of the gold samples with and without the organic overlayer, respectively.

\section{Results and discussion}

\subsection{Contact angles and ellipsometry}

Effect of chain length difference. Contact angles, advancing and receding, of water and hexadecane, and ellipsometric thicknesses were recorded for the gradient assemblies formed from alkanethiols of different chain lengths. Advancing and receding contact angles with water are shown in Fig. 2 for a $\mathrm{HS}\left(\mathrm{CH}_{2}\right)_{11}-\mathrm{CH}_{3} /$ $\mathrm{HS}-\left(\mathrm{CH}_{2}\right)_{17}-\mathrm{CH}_{3} \quad(\mathrm{C} 12 / \mathrm{C} 18)$ gradient. The shorter alkanethiol is on the left side (diffuses from the left) and the longer is on the right side (diffuses from the right). This convention is followed throughout all figures. On the $\mathrm{C} 12$-side (left) the contact angles, $\theta_{\mathrm{a}}=106^{\circ}$ and $\theta_{\mathrm{r}}=100^{\circ}$, differs slightly from the $\theta_{\mathrm{a}} \sim 110^{\circ}$ and $\theta_{\mathrm{r}} \sim 105^{\circ}$ 


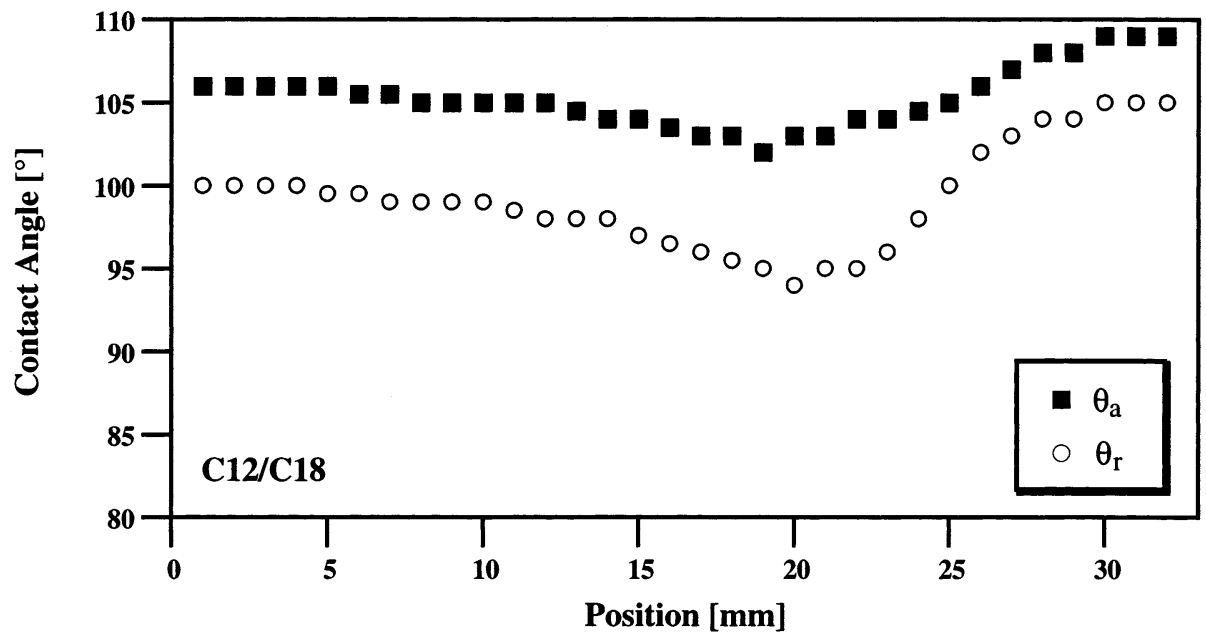

Fig. 2. Advancing and receding contact angle with water, and hysteresis for a C14/C18 gradient. The shorter alkanethiol is on the left side (diffusses from the left) and the longer is on the right side (diffusses from the right). This convention is followed throughout all figures.

normally seen for crystalline $\mathrm{CH}_{3}$ surfaces indicating a partial disorder and $\mathrm{CH}_{2}$ exposure of the outermost portion of the SAM. This exposure increases as we move along the gradient, to reach a maximum, and a corresponding minimum in contact angles of $\theta_{\mathrm{a}}=103^{\circ}$ and $\theta_{\mathrm{r}}=95^{\circ}$, at around $19-20 \mathrm{~mm}$. This is in the mixed region of the assembly and the results indicate a substantial amount of $\mathrm{CH}_{2}$ exposure "polyethylene like". After this minimum the contact angles increase to $\theta_{\mathrm{a}}=109^{\circ}$ and $\theta_{\mathrm{r}}=103^{\circ}$ on the far C18-side (right). These values indicate that an ordered crystalline structure is formed, analogous to that formed by assembly from a single component $n$-alkanethiol solution [27,28]. Moreover, no stickand-slip behavior is observed as the liquid drop moves along the surface. Thus, the employed cleaning procedure appears to be very effective in removing surface contamination, e.g. remaining fragments or aggregates of the Sephadex matrix.

Fig. 3(a-d) shows the advancing and receding contact angles with hexadecane for the following four diffusion pairs: (a) $\mathrm{HS}-\left(\mathrm{CH}_{2}\right)_{15}-\mathrm{CH}_{3} / \mathrm{HS}-$ $\left(\mathrm{CH}_{2}\right)_{17}-\mathrm{CH}_{3}(\mathrm{Cl} / \mathrm{Cl})$; (b) $\mathrm{HS}-\left(\mathrm{CH}_{2}\right)_{13}-\mathrm{CH}_{3} /$ $\mathrm{HS}-\left(\mathrm{CH}_{2}\right)_{17}-\mathrm{CH}_{3}(\mathrm{C} 14 / \mathrm{Cl})$; (c) $\mathrm{HS}-\left(\mathrm{CH}_{2}\right)_{11}-$ $\mathrm{CH}_{3} / \mathrm{HS}-\left(\mathrm{CH}_{2}\right)_{17}-\mathrm{CH}_{3}(\mathrm{C} 12 / \mathrm{C} 18)$ and (d) HS$\left(\mathrm{CH}_{2}\right)_{9}-\mathrm{CH}_{3} / \mathrm{HS}-\left(\mathrm{CH}_{2}\right)_{17}-\mathrm{CH}_{3}(\mathrm{Cl} / \mathrm{Cl})$. The curves are representative candidates of several equally prepared gradients. From the figures it can be seen that the gradient region appears around 15-20 $\mathrm{mm}$ for all diffusion pairs as probed with the contact angle of hexadecane. This value of the length of the gradient region is larger than what has been reported in preceding publications [1,2]. One reason for this difference could be that we for the first time use contact angles as a mean to characterize the whole gradient. Contact angles have proven to be very sensitive to the chemical composition even on the microscopic level (although the contact angle reading is the sum of all the interactions affecting the drop). Another contributing effect that has to be considered is that we have altered the diffusion/adsorption conditions [1]. Larger amounts of thiol solution have been injected into the filters and a longer diffusion/adsorption time has been employed (ca. $65 \mathrm{~h}$ ). The larger amounts of thiol molecules allow for the diffusion fronts to interdiffuse to a greater extent, as well as the longer incubation times do, leading to a larger probability for exchange reactions to occur. The longer incubation times might, on the other hand, give room for more phase segregation. We also observe an asymmetric shape of the contact angle curves, where the slope is always smaller on the side with the shorter alkanethiol, Fig. 3(a-d). 

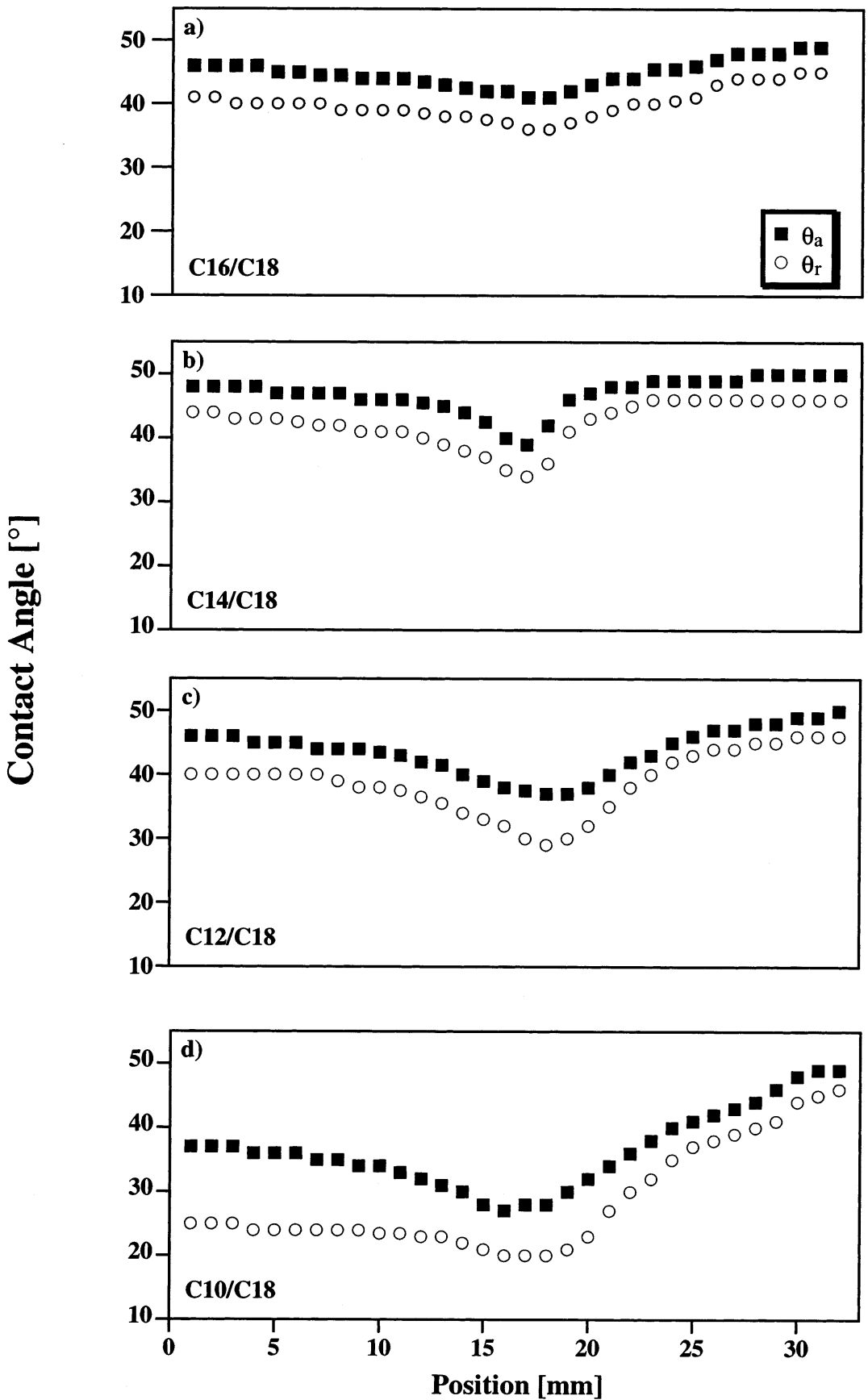

Fig. 3. Advancing and receding contact angles of hexadecane for the different gradients. (a) $\mathrm{The} \mathrm{C} 16 / \mathrm{Cl} 8\left(\mathrm{HS}\left(\mathrm{CH}_{2}\right)_{15} \mathrm{CH}_{3} /\right.$ $\left.\mathrm{HS}\left(\mathrm{CH}_{2}\right)_{17} \mathrm{CH}_{3}\right)$ gradient; (b) the $\mathrm{Cl} / \mathrm{Cl} \quad\left(\mathrm{HS}\left(\mathrm{CH}_{2}\right)_{13} \mathrm{CH}_{3} / \mathrm{HS}\left(\mathrm{CH}_{2}\right)_{17} \mathrm{CH}_{3}\right)$ gradient; (c) the $\mathrm{Cl2} / \mathrm{Cl} \quad\left(\mathrm{HS}\left(\mathrm{CH}_{2}\right)_{11} \mathrm{CH}_{3} /\right.$ $\left.\mathrm{HS}\left(\mathrm{CH}_{2}\right)_{17} \mathrm{CH}_{3}\right)$ gradient and (d) the $\mathrm{C} 10 / \mathrm{Cl}\left(\mathrm{HS}\left(\mathrm{CH}_{2}\right)_{9} \mathrm{CH}_{3} / \mathrm{HS}\left(\mathrm{CH}_{2}\right)_{17} \mathrm{CH}_{3}\right)$ gradient. 
This feature is probably due to a competition between the quicker diffusion of the shorter alkanethiol with respect to the longer and the exchange of the shorter alkanethiol by the longer. The minimum is not likely to occur at 50:50 ratio of short/long alkanethiol on the surface. It would rather be at a surface composition were the short alkanethiol is dominant, as indicated by the results of Bain et al. [18]. For the extreme ends of each gradient type, Fig. 3(a-d), the resulting values approach the typical values of a well ordered $\mathrm{CH}_{3}$-terminated monolayer, $\theta_{\mathrm{a}} \sim 45-50^{\circ}$ and $\theta_{\mathrm{r}} \sim 40-46^{\circ}$, except for the C10-side (Fig. 3(d)) $[16,18,28,29]$. At the $\mathrm{C} 18$-ends of the gradients we observe how the values reach $\theta_{\mathrm{a}} \sim 49-50^{\circ}$ and $\theta_{\mathrm{r}} \sim 45-46^{\circ}$. For $\mathrm{C} 10$ (in the $\mathrm{C} 10 / \mathrm{C} 18$ gradient, see Fig. 3(d)) on the other hand, we start at values of $\theta_{\mathrm{a}} \sim 37^{\circ}$ and $\theta_{\mathrm{r}} \sim 25^{\circ}$, which again tells us that this part of the gradient is not perfectly ordered. This amount of disorder was not expected, and could be interpreted to indicate the ordering process in this part of the assembly is very time consuming and is not completed in the ca. $65 \mathrm{~h}$ used here. Another possible explanation is that the longer thiols continuously replace shorter ones on the $\mathrm{C} 10$ side. The lowest contact angles observed along the gradients are for $\mathrm{C} 16 / \mathrm{C} 18: \theta_{\mathrm{a}} \sim$ $41^{\circ}$ and $\theta_{\mathrm{r}} \sim 36^{\circ}, \mathrm{C} 14 / \mathrm{C} 18: \theta_{\mathrm{a}} \sim 39^{\circ}$ and $\theta_{\mathrm{r}} \sim 34^{\circ}$, C12/C18: $\theta_{\mathrm{a}} \sim 37^{\circ}$ and $\theta_{\mathrm{r}} \sim 30^{\circ}$, and $\mathrm{C} 10 / \mathrm{C} 18$ : $\theta_{\mathrm{a}} \sim 28^{\circ}$ and $\theta_{\mathrm{r}} \sim 20^{\circ}$. All values are indicative of various amounts of disorder or $\mathrm{CH}_{2}$-exposure and we see an increase with increasing difference in chain length as expected. What is surprising though, is the small hysteresis $\left(\theta_{\mathrm{a}}-\theta_{\mathrm{r}}\right)$ that is seen in the middle of the gradients (around 17-20 $\mathrm{mm})$. For $\mathrm{C} 16 / \mathrm{C} 18: \theta_{\mathrm{a}}-\theta_{\mathrm{r}}=7^{\circ}, \mathrm{C} 14 / \mathrm{C} 18: \theta_{\mathrm{a}}-$ $\theta_{\mathrm{r}}=5^{\circ}, \mathrm{C} 12 / \mathrm{C} 18: \theta_{\mathrm{a}}-\theta_{\mathrm{r}}=7^{\circ}$, and $\mathrm{C} 10 / \mathrm{C} 18$ : $\theta_{\mathrm{a}}-\theta_{\mathrm{r}}=8^{\circ}$. No clear trend can be observed for the differences in chain lengths. Drelich et al. observed a small hysteresis for water of single component SAMs of dodecanethiol on gold [29]. Chaudhury et al. also reported a low hysteresis $\left(10-6^{\circ}\right)$ for water on gradients prepared from vapor diffusion of decyltrichlorosilane [30]. This indicates that the origin of the hysteresis is microscopic, and not macroscopic. Thus, it is more likely that it is surface chemistry rather than surface roughness that determines the hysteresis for these surface assemblies. On the $\mathrm{C} 10 / \mathrm{C} 18$-gradient though, we can observe how the hysteresis increases (up to $22^{\circ}$ ) as we move towards the C10-end (to the left in Fig. 3(d)). This is consistent with the observation (see above) that we have a large amount of disorder in that part of the gradient, rendering the surface more heterogeneous.

In Fig. 4(a-d) the variations in ellipsometric thicknesses of the $\mathrm{C} 16 / \mathrm{C} 18$ (Fig. 4(a)), C14/C18 (Fig. 4(b)), C12/C18 (Fig. 4(c)) and C10/C18 (Fig. 4(d)) are shown. The C18-side has a thickness of $22 \pm 2 \AA$, and for $\mathrm{C} 10, \mathrm{C} 12, \mathrm{C} 14$ and $\mathrm{C} 16$ we observe thicknesses of $11 \pm 1,14 \pm 1,16 \pm 1$ and $19 \pm 1 \AA$, respectively. This agrees well with simple space-filling models of $n$-alkanethiolate assemblies in general and with experimentally observed thicknesses [27,28,31]. The $\mathrm{C} 16 / \mathrm{C} 18$ (Fig. 4(a)) gradient displays a smooth and slow transition from ca. 19 to $22 \AA$ across a distance of approximately $15 \mathrm{~mm}$. The similar diffusion rates and the probably slow exchange rate of these molecules is likely to accommodate for good intermixing resulting in the smooth behavior. For both the C14/C18 (Fig. 4(b)) and the C12/C18 (Fig. 4(c)) the transition is more abrupt and occurs within ca. $10 \mathrm{~mm}$. Their behavior is quite similar, indicating that the processes involved strongly resemble each other. Finally, the $\mathrm{C} 10 / \mathrm{C} 18$ (Fig. 4(d)) gradient exhibits a slower change from short to long chain alkanethiol, around 15-17 $\mathrm{mm}$. The C10 diffuses faster than the $\mathrm{C} 18$ and therefore reach further into the $\mathrm{C} 18$-side, but the exchange process should counteract this. The time scales for the exchange processes in these assemblies are not yet known, though. Comparing the position of the onset of the thickness change with the position of the minimum contact angle of the gradients, further supports the perception that the greatest disorder is achieved when the amount of $\mathrm{C} 18$ on the surface is low. A quick estimation using a linear relationship for thicknesses (setting the thickness of C14: $16 \AA$ and C18: $22 \AA$ ) in the monolayer assembly for the $\mathrm{C} 14 / \mathrm{C} 18$ gradient reveals that the contact angle minimum coincides roughly with a surface composition of $\mathrm{C} 14$ ca. $80 \%$ and $\mathrm{C} 18$ ca. $20 \%$. A sparse distribution of $\mathrm{C} 18$ chains in a sea of $\mathrm{C} 14$ chains would allow for 

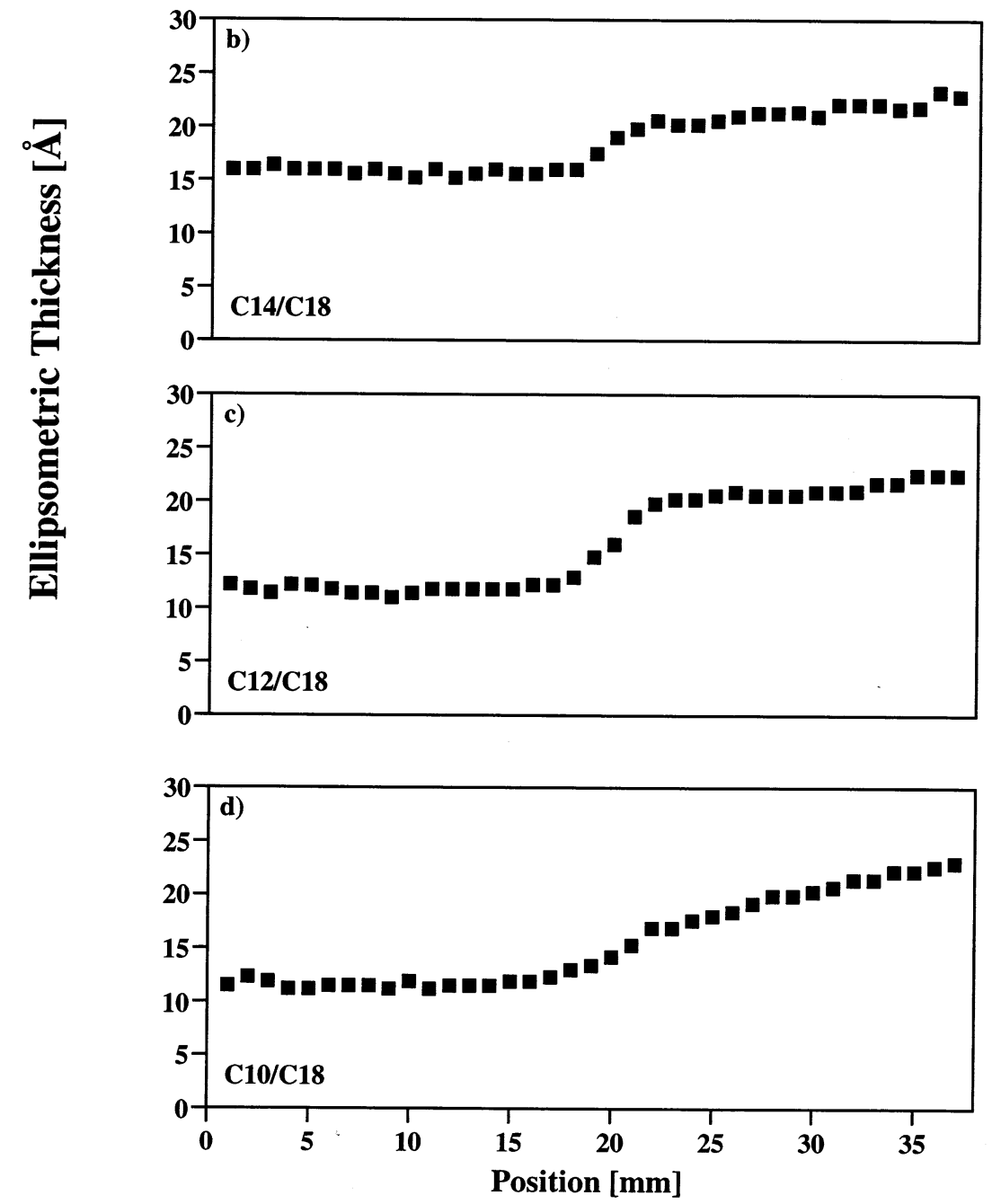

Fig. 4. Ellipsometric thicknesses for (a) the $\mathrm{C} 16 / \mathrm{C} 18$ gradient; (b) the $\mathrm{C} 14 / \mathrm{C} 18$ gradient; (c) the $\mathrm{C} 12 / \mathrm{C} 18$ gradient and (d) the C10/C18 gradient.

a fold-over of the longer chains on top of the shorter chains, resulting in a maximum exposure of $\mathrm{CH}_{2}$ groups. The validity of this estimation can be questioned though, since it has not been conclusively shown that the refractive index of the SAM is the same for all chain conformations and 
all chain lengths. The estimation is merely intended to serve as a hint, and further experimental work has to be conducted to reveal the true surface composition.

\subsection{Effect of formation time}

Given the importance of the time allowed for gradient formation, a third point of interest is the kinetics involved. We have collected contact angles and ellipsometric data for three adsorption/ diffusion times to show some kinetic features of the assembly processes.

In Fig. 5(a-d), the advancing contact angle (Fig. 5(a)), the receding contact angle (Fig. 5(b)), the hysteresis, $\theta_{\mathrm{a}}-\theta_{\mathrm{r}}$, (Fig. 5(c)), and ellipsometric thicknesses (Fig. 5(d)) are shown for three different adsorption/diffusion times 50, 65 and $97 \mathrm{~h}$, respectively, for the assembly of a $\mathrm{C} 14 / \mathrm{C} 18$ gradient. For $\theta_{\mathrm{a}}$ and $\theta_{\mathrm{r}}$ (Fig. 5(a and b)) a number of features change with time. First, at the far C14side, it is not until the $65 \mathrm{~h}$ sample that the contact angles reach the high and stable values of a ordered crystalline $\mathrm{CH}_{3}$-terminated monolayer. Second, the contact angles decrease slower and on a longer stretch when going to the middle from the C14-side, as compared to going from the C18-side. This could be indicative of a slower ordering process. The slower ordering process might be expected if one consider that the energy gained from forming a well-ordered C14 monolayer is smaller than for a $\mathrm{C} 18$ monolayer, i.e. the van der Waals contribution per methylene unit. On the C18-side, the contact angles start at high values $\left(\theta_{\mathrm{a}}=50^{\circ}\right.$ and $\left.\theta_{\mathrm{r}}=46^{\circ}\right)$ that are maintained for ca. $10 \mathrm{~mm}$, after which they sink abruptly as we move to the left along the gradient. The minimum values for $\theta_{\mathrm{a}}$ and $\theta_{\mathrm{r}}$ change with time as well. This could originate both from the ordering process and from the exchange process of short to long chains in the intermixing region. In Fig. 5(c), it is observed how the hysteresis $\left(\theta_{\mathrm{a}}-\theta_{\mathrm{r}}\right)$ is subject to change with time as it decreases with increasing adsorption/diffusion times. The ellipsometry results (Fig. 5(d)) show the incompleteness in coverage(thickness) of the $50 \mathrm{~h}$ gradient as compared to the 65 and $97 \mathrm{~h}$ samples. On the C14-side, the thickness is below $15 \AA$ whereas on the $\mathrm{C} 18$-side it agrees quite well with a single component C18 SAM already at $50 \mathrm{~h}$. It is at present hard to tell whether it is the diffusion or the ordering process, or a combination of both, that is responsible for the gradient formation.

\section{IRAS characterization versus contact angle}

Fig. 6 shows the IRAS spectra for a $\mathrm{C} 12 / \mathrm{C} 18$ gradient in the high frequency region where the hydrocarbon stretching group frequencies are expected to absorb. The asymmetric and symmetric $\mathrm{CH}_{2}$ and $\mathrm{CH}_{3}$ vibrations are clearly visible [3234]. All four peaks are sensitive to changes in the environment, which shift the mode frequencies and change their intensities. This is most evident for the $\mathrm{CH}_{2}$ peaks, and in particular the asymmetric stretch $\left(\mathrm{d}^{-}\right)$at around $2920 \mathrm{~cm}^{-1}$. Moving from the $\mathrm{C} 12$-end to the $\mathrm{C} 18$-end, the peak position shifts, the intensity increases, and the band shape is narrowed. This is expected since the C18 part of the gradient should be more ordered, thus shifting the peak to lower wavenumbers. The increasing order should also narrow the band, and more methylene units give a higher intensity. This is indeed observed. In Fig. 7( $\mathrm{a}-\mathrm{c})$, we have focused our attention to the $\mathrm{d}^{-}$-peak. The position of the peak and its full width at half maximum (FWHM) are shown in Fig. 7(a-b) for the C12/ $\mathrm{C} 18$ and the $\mathrm{C} 16 / \mathrm{C} 18$ gradients. For the $\mathrm{C} 12 / \mathrm{C} 18$ gradient, the position of the $\mathrm{d}^{-}$-peak (Fig. 7(a)) starts at ca. $2922 \mathrm{~cm}^{-1}$ on the C12-side. It shifts to $2924 \mathrm{~cm}^{-1}$ after $6 \mathrm{~mm}$, after which it slowly decreases to $2919-20 \mathrm{~cm}^{-1}$ on the C18-side. The position of the $\mathrm{d}^{-}$-peak for the $\mathrm{C} 16 / \mathrm{C} 18$ gradient behaves differently. It starts at $2918 \mathrm{~cm}^{-1}$ on the C16-side, increases to ca. $2920 \mathrm{~cm}^{-1}$ at $4 \mathrm{~mm}$ and goes slowly back to $2917-18 \mathrm{~cm}^{-1}$ on the C18side. The peak positions $\left(\mathrm{d}^{-}\right)$of the $\mathrm{C} 12 / \mathrm{C} 18$ gradient are not identical to those found for well ordered single component alkanethiolate SAMs on gold, where $\mathrm{d}^{-}$normally is observed at 2918 $\mathrm{cm}^{-1}$. Instead there is a certain amount of disorder. The monitoring of the FWHM of the $\mathrm{d}^{-}$peak for the $\mathrm{C} 12 / \mathrm{C} 18$ gradients lends further support to this perception, as it does not sink down to $14 \mathrm{~cm}^{-1}$ until the very end on the 

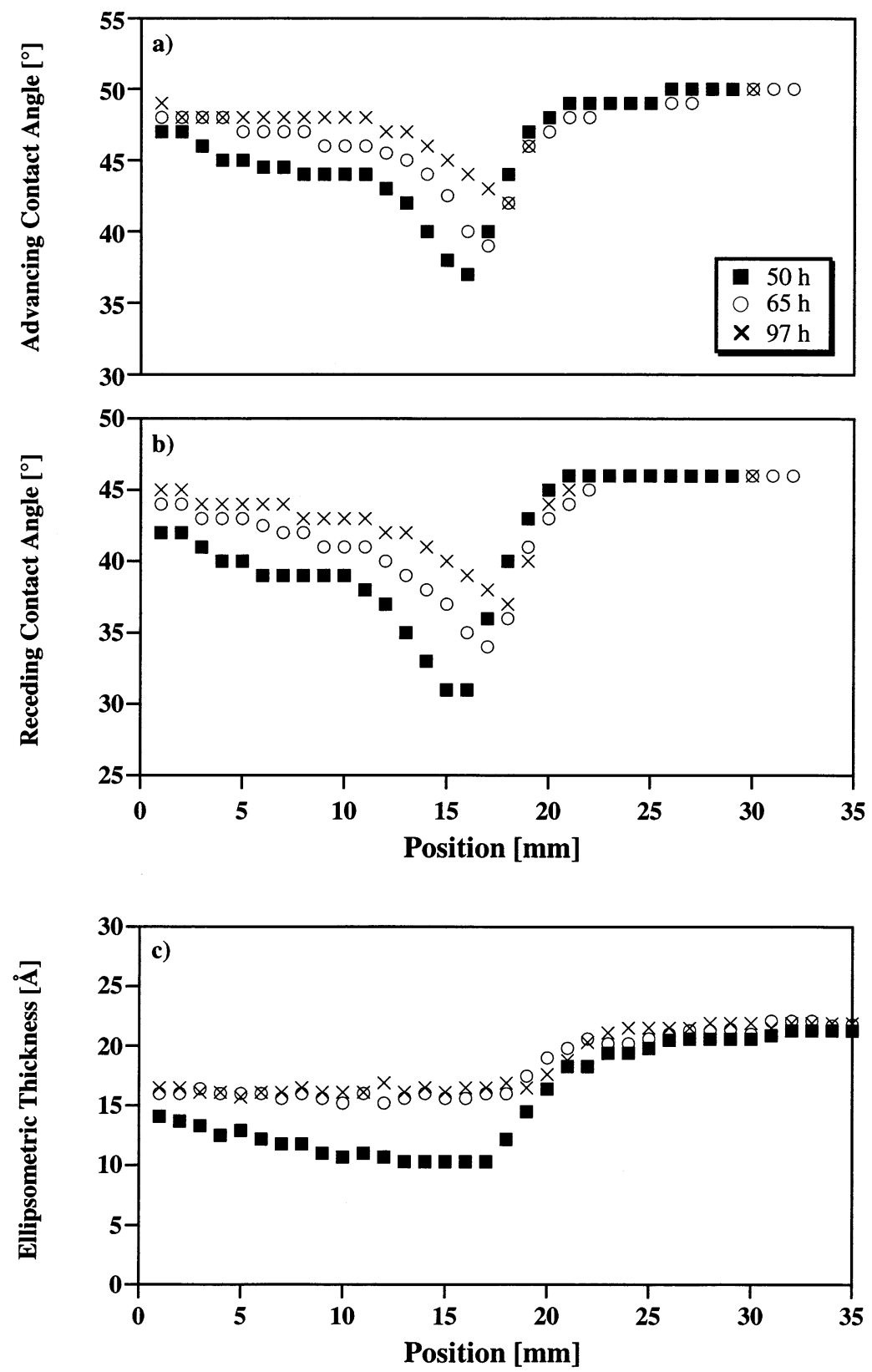

Fig. 5. Time evolution of the adsorption/diffusion process of a C14/C18 gradient. (a) Advancing contact angles with hexadecane; (b) receding contact angles with hexadecane and (c) ellipsometric thicknesses, for adsorption/diffusion times of 50 , 65 and $97 \mathrm{~h}$.

C18-side (Fig. 7(b)). Normal values for a single component alkanethiolate SAM would be FWHM ca. $11-12 \mathrm{~cm}^{-1}$ [22,31]. The C16/C18 gradient is more ordered, and the FWHM (Fig. 7(b) goes from 13 up to $15 \mathrm{~cm}^{-1}$ and finally down to $12 \mathrm{~cm}^{-1}$ as we scan along the gradient. The gradient surfaces prepared for IRAS measurements are shorter (30 $\mathrm{mm}$ instead of $40 \mathrm{~mm}$ ), which means that the glass filters of the diffusion setup are closer together. The same amount (600 


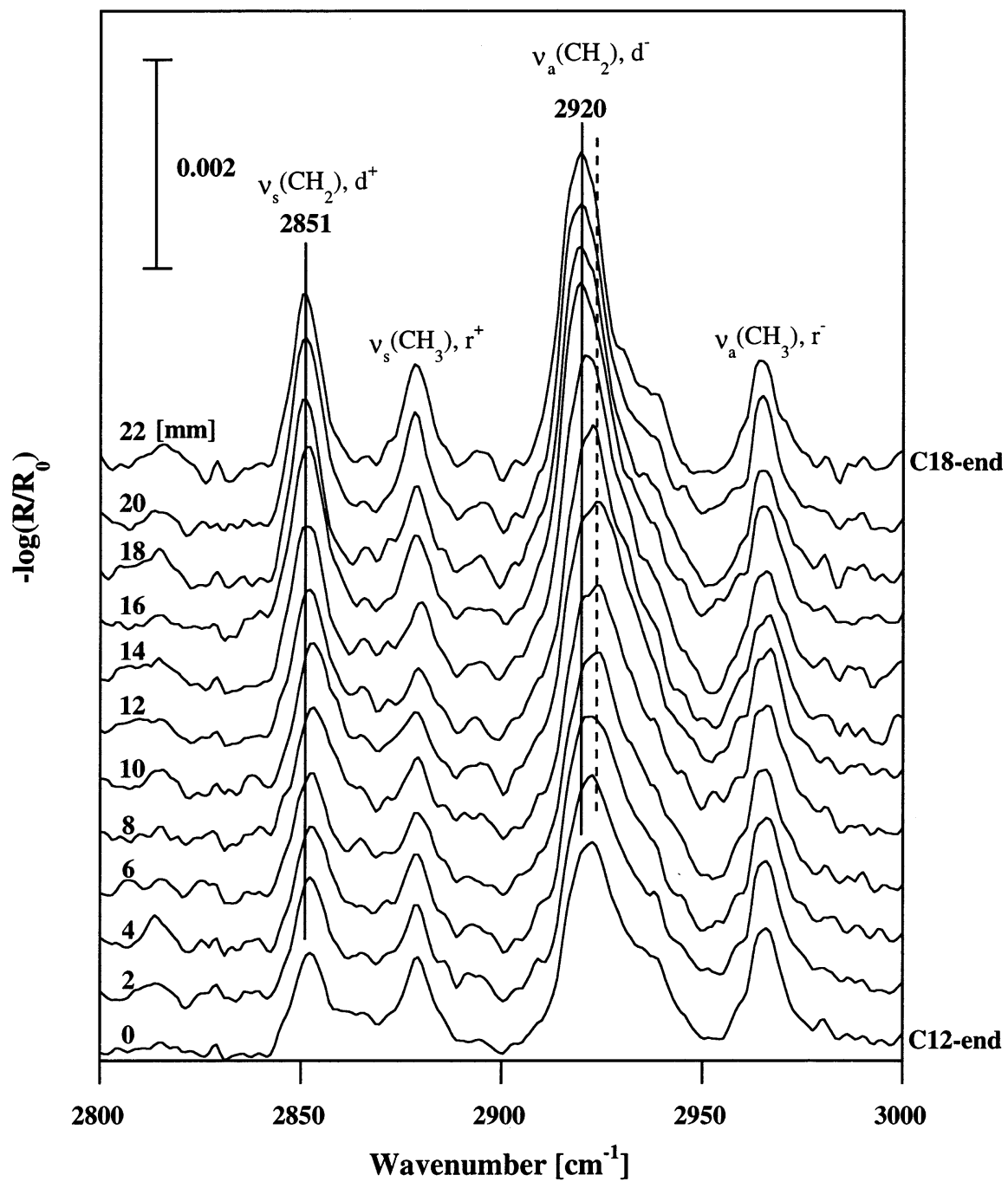

Fig. 6. IRAS spectra for a $\mathrm{C} 12 / \mathrm{C} 18$ gradient in the high frequency region, $2800-3000 \mathrm{~cm}^{-1}$. Peak assignments are indicated. The step length along the gradient between each spectrum was $2 \mathrm{~mm}$.

$\mu 1)$ of thiol solutions have been pipetted into the filters though. This might cause greater intermixing during the diffusion due to lesser concentration depletion in the matrix as the thiol diffusion fronts move through the matrix, as compared to the surfaces prepared exclusively for ellipsometry and contact angles. From the data in Fig. 7(a-b), we again note that the ordering process for the longer chains $(\mathrm{C} 16$ and $\mathrm{C} 18)$ is faster than that of the shorter chain (C12).

Finally, an attempt to correlate the contact angles (advancing and receding contact angles for hexadecane) with the position of the $\mathrm{d}^{-}$-peak was made, see Fig. 7(c), for a $\mathrm{C} 16 / \mathrm{C} 18$ gradient. It should be pointed out that the gradient prepared for contact angle measurements and the one for IRAS measurement, both were formed on $30 \mathrm{~mm}$ long gold substrates to facilitate for the comparison. The uncertainty in the correlation of the position is estimated to $1 \mathrm{~mm}$ between the contact angle and IRAS measurements. The end points of the gradient display values of 2918 to $2917 \mathrm{~cm}^{-1}$ 

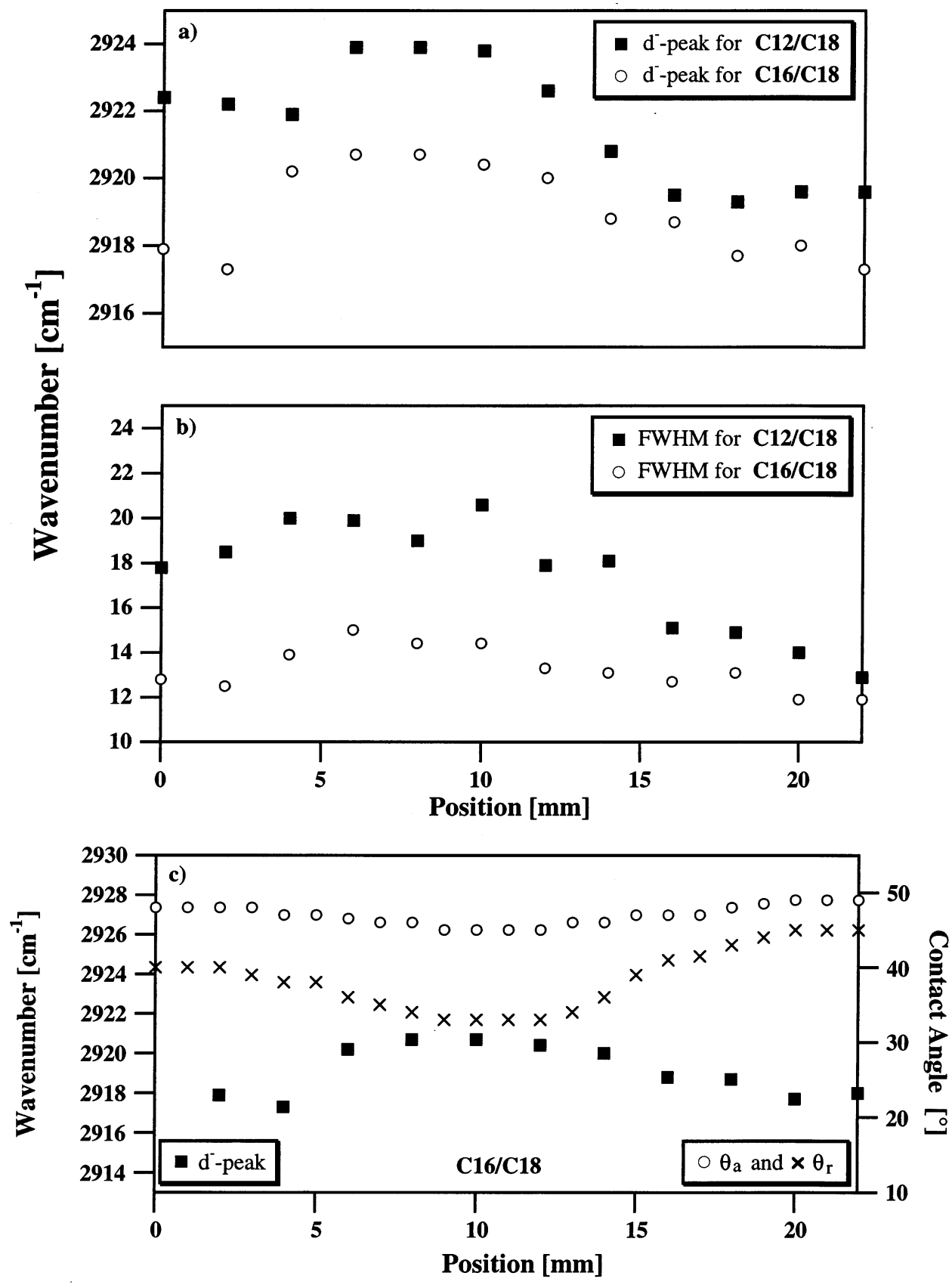

Fig. 7. (a) Peak position of the $\mathrm{d}^{-}$vibration of a $\mathrm{C} 12 / \mathrm{C} 18$ and a $\mathrm{C} 16 / \mathrm{C} 18$ gradient, (b) FWHM values for a $\mathrm{C} 12 / \mathrm{C} 18$ and a $\mathrm{C} 16 / \mathrm{C} 18$ gradient and (c) correlation between the advancing and receding contact angle of hexadecane, and the position of the $\mathrm{d}^{-}$ peak for the $\mathrm{C} 16 / \mathrm{C} 18$ gradient. Observe that the peak position data in (c) has been shifted $2 \mathrm{~mm}$ to the right to coincide with the contact angle data. 
for the $\mathrm{d}^{-}$-peak together with advancing contact angles of hexadecane equal to 48 and $49^{\circ}$ for the $\mathrm{C} 16$ and the $\mathrm{C} 18$ sides, respectively. The middle part of the gradient, 6-14 $\mathrm{mm}$, displays some disorder as interpreted by the shift of the $\mathrm{d}^{-}$-peak from 2918 to $2921 \mathrm{~cm}^{-1}$. $\theta_{\text {a }}$ does support this observation as it goes down from $49-48^{\circ}$ to $44^{\circ}$, and $\theta_{\mathrm{r}}$ suggests that some heterogeneity is present in the monolayer assembly as it drops down to $34^{\circ}$ in the middle, compare the $45-40^{\circ}$ at the ends. The increased hysteresis presented by this gradient as compared to gradients prepared on longer gold substrates (Fig. 3(a)), could be a result of the greater interdiffusion due to a shorter distance between the filters. That should result in a better mixing on the surface over a greater distance, but since there is only two methylene units difference between $\mathrm{C} 16$ and $\mathrm{C} 18$ we can still observe high advancing contact angles. Thus, the structural observations correlate well between IRAS and contact angle measurements.

\section{Summary}

It is feasible to prepare gradient assemblies by cross diffusion of two alkanethiols of different chain lengths on gold, thus achieving continuously varying properties in terms of order/disorder on a surface. Contact angles, ellipsometry and IRAS have been employed to demonstrate some of the structural properties of the SAM gradients. With contact angles, it is seen that the gradient region spans $15-20 \mathrm{~mm}$ in length for assemblies with chain length differences of $8,4,6$ and 2 methylene groups. The contact angle measurements have an inherently high sensitivity to chemical composition. IRAS provides vital information about the internal structure of the monolayer, and provides evidence for the presence of order/disorder (trans or gauche conformers) amongst the hydrocarbon chains. Ellipsometry gives quick and reliable information about monolayer thicknesses along the gradient, which can serve as a reference in comparison to single component monolayers. The different methods complement each other and offer a more complete picture of the properties of the gradients. All results taken into consid- eration, it is likely that there is not a perfect mixing at the molecular level in these gradient assemblies. The fact that we do not observe zero contact angles using hexadecane, and that we have a certain hysteresis for all the gradients indicate a heterogeneity on some level. A maximum chain length difference of eight methylene groups is perhaps not enough to allow for only $\mathrm{CH}_{2}$ exposure at perfect mixing. The small hysteresis that is observed indicates good intermixing, though. It lies close at hand, to believe that the heterogeneity is on a small length scale. From these measurements it is also clear that the formation mechanisms of the assemblies are complex. Different length of the alkanethiols used give differences in diffusion rates, the ordering processes and the exchange process. A great deal of interesting subjects remain to be studied that concern these gradient assemblies. The true surface coverage is of course essential to establish, but also to describe the diffusion of the alkanethiols in the matrix to understand the influence of 'solution' concentration. Further investigation of the ordering and exchange processes are also of great interest. These gradient assemblies provide a unique possibility to control and investigate different aspects of mobility and steric hindrance during adsorption of biomolecules onto solid surfaces.

\section{Acknowledgements}

This research project was supported by the Swedish Research Council for Engineering Sciences (TFR), the Swedish National Board for Technical and Industrial Development (NUTEK) and the Swedish Natural Science Research Council (NFR) through the Swedish Biomaterials Consortium.

\section{References}

[1] B. Liedberg, P. Tengvall, Langmuir 11 (1995) 3821-3827.

[2] B. Liedberg, M. Wirde, Y.-T. Tao, P. Tengvall, U. Gelius, Langmuir 13 (1997) 5329-5334.

[3] A.S. Hoffman, Ann. New York Acad. Sci. 516 (1987) 96-101. 
[4] B.D.J. Ratner, Biomed. Mater. Res. 27 (1993) 837-850.

[5] S. Löfås, B. Johnsson, J. Chem. Comm. Chem. Soc. 1990, 1256-1258.

[6] A. Ulman, An Introduction to ultrathin organic films. From Langmuir-Blodgett to Self-Assembly, 1st edition, Academic Press, San Diego, 1991.

[7] K.D. Schierbaum, T. Weiss, E.U. Thoden van Velzen, et al., Science 265 (1994) 1413-1415.

[8] L.-H. Lee, in: L.-H. Lee (Ed.), Fundamentals of Adhesion, Plenum Press, New York, 1991, p. 454.

[9] K.L. Prime, G.M. Whitesides, Science 252 (1991) 11641167.

[10] K.L. Prime, G.M. Whitesides, J. Am. Chem. Soc. 115 (1993) 10714-10721.

[11] C.N. Sukenik, N. Balachander, L.A. Culp, K. Lewandowska, K. Merritt, J. Biomed. Mater. Res. 24 (1990) 1307-1323.

[12] S. Margel, E.A. Vogler, L. Firment, T. Watt, S. Haynie, D.Y. Sogah, J. Biomed. Mater. Res. 27 (1993) 14631476.

[13] M. Lestelius, in: M. Lestelius (Ed.), Plasma Protein Adsorption on to Thiol Modified Gold Surfaces, Linköping Univeristy, Sweden, 1995.

[14] C.B. Herbert, T.L. McLernon, C.L. Hypolite, et al., Chem. Biol. 4 (1997) 731-737.

[15] C.L. Hypolite, T.L. McLernon, D.N. Adams, et al., Bioconjugate Chem. 8 (1997) 658-663.

[16] P.E. Laibinis, R.G. Nuzzo, G.M. Whitesides, J. Phys. Chem. 96 (1992) 5097-5105.

[17] J.P. Folkers, P.E. Laibinis, G.M. Whitesides, J. Deutch, J. Phys. Chem. 98 (1994) 563-571.

[18] C.D. Bain, G.M. Whitesides, J. Am. Chem. Soc. 111 (1989) 7164-7175.
[19] S.J. Stranick, A.N. Parikh, Y.-T. Tao, D.L. Allara, P.S. Weiss, J. Phys. Chem. 98 (1994) 7636-7646.

[20] J.B. Schlenoff, M. Li, H. Ly, J. Am. Chem. Soc. 117 (1995) 12528-12536.

[21] R.J. Good, in: R.J. Good (Ed.), Contact Angle, Wetting, and Adhesion: A Critical Review, 1st edition, VSP, Utrecht, 1993, p. 971.

[22] S.V. Atre, B. Liedberg, D.L. Allara, Langmuir 11 (1995) 3882-3893.

[23] M. Lestelius, P. Tengvall, I. Lundström, J. Colloid Interface Sci. 171 (1995) 533-535.

[24] J. Shi, B. Hong, A.N. Parikh, R.W. Collins, D.L. Allara, Chem. Phys. Lett. 246 (1995) 90-94.

[25] I. Engquist, I. Lundström, B. Liedberg, J. Phys. Chem. 99 (1995) 12257-12267.

[26] I. Engquist, M. Lestelius, B. Liedberg, J. Phys. Chem. 99 (1995) 14198-14200.

[27] C.D. Bain, E.B. Troughton, Y.-T. Tao, J. Evall, G.M. Whitesides, R.G. Nuzzo, J. Am. Chem. Soc. 111 (1989) $321-335$.

[28] P.E. Laibinis, G.M. Whitesides, D.L. Allara, et al., J. Am. Chem. Soc. 113 (1991) 7152-7167.

[29] J. Drelich, J.D. Miller, R.J. Good, J Colloid Interface Sci. 179 (1996) 37-50.

[30] M.K. Chaudhury, G.M. Whitesides, Science 256 (1992) 1539-1541.

[31] M.D. Porter, T.B. Bright, D.L. Allara, C.E.D. Chidsey, J. Am. Chem. Soc. 109 (1987) 3559-3568.

[32] R.G. Snyder, S.L. Hsu, S. Krimm, Spectrochim. Acta 34A (1978) 395-406.

[33] R.G. Snyder, H.L. Strauss, C.A. Elliger, J. Phys. Chem. 86 (1982) 5145-5150.

[34] R.A. MacPhail, H.L. Strauss, R.G. Snyder, C.A. Elliger, J. Phys. Chem. 88 (1984) 334-341. 